\title{
Deposited BSA/lgG Conjugated Up-Conversion Nanoparticles by the Matrix-Assisted Pulsed Laser Evaporation (MAPLE)
}

\author{
Songlin Yang, Jin Zhang \\ University of Western Ontario \\ London, Ontario, Canada, N6A 5B9 \\ jzhang@eng.uwo.ca; syang368@uwo.ca
}

\begin{abstract}
In this paper, the up-conversion nanoparticles were synthesized through chemical method and conjugated with bovine serum albumin (BSA)/ Immunoglobulin G (IgG) through different method. The up-conversion nanoparticles, BSA conjugated upconversion nanoparticles and $\mathrm{IgG}$ conjugated up-conversion nanoparticles were deposited by matrix-assisted pulsed laser evaporation (MAPLE) technique. The conjugation/deposition result has been investigated by Transmission electron microscopy (TEM) and Fourier transform infrared spectroscopy (FT-IR). The result of this study demonstrates that MAPLE is a suitable process for depositing organic/biological nanoparticles and the up-conversion nanoparticles could be deposited through MAPLE technique.
\end{abstract}

Keywords: Up-conversion nanoparticles, Matrix-assisted pulsed laser evaporation, Immunoglobulin G, Bovine serum albumin, Proteins, Fourier transform infrared spectroscopy

\section{Introduction}

Lanthanide-doped up-conversion nanoparticles have been developed and studied by researchers for decades. The upconversion nanoparticles is famous for holding the non-linear optical property known as anti-Stokes emission. This property means that the particles could absorb two or more low-energy photons and result in high-energy luminescent emission[1-3]. The up-conversion nanoparticles usually contain rare-earth elements (Thulium, Gadolinium, Erbium etc.). Since the up-conversion nanoparticles possess anti-Stokes property, the particles could be excited by NIR light and emit UV-visible light[4]. Besides, this process could active photosensitizers which conjugated on their surface through resonance energy transfer and generate ROS or reacting $\mathrm{O}_{2}$ to eliminate cancer cells[5-7]. For past fifty years, the researchers made enormous progress on developing the application of up-conversion nanoparticles, especially in the bioapplication field. The no-linear property of up-conversion nanoparticles could be applied in vitro cell and tissue imaging[8], fluorescence diffuse optical tomography [9] and photo-thermal agents for cancer cell treatment[10].

Matrix assisted pulsed laser evaporation (MAPLE) technique is a surface modification method which was developed from the pulse laser deposition (PLD) by the Naval Research Laboratories in mid 1980s[11]. This technique was developed to overcome the drawback of the conventional PLD technique which need the target material to expose at the harsh conditions. By dissolving the target material in the solvent and froze by liquid nitrogen, MAPLE technique could deposit organic materials, biomaterials and proteins[12]. Meanwhile, the influence of the high energy laser beam is minimized since most of the laser energy is absorbed by the frozen target solution. In this method, the target material is normally dissolved or suspended in solvent which is highly volatile. The solution is then frozen by liquid nitrogen. And the frozen solution (target) is irradiated by the pulsed laser beam. This whole process is performed under vacuum $\left(1 \times 10^{-6}\right.$ Torr $)$. Solvent molecules are pumped away. And the target molecules which is heavier than the solvent molecules are deposited on the surface of the substrate.

In this study, the BSA/IgG proteins were conjugated with up-conversion nanoparticles and deposited through MAPLE techniques. The Fourier transform infrared spectroscopy (FT-IR) and Transmission electron microscopy (TEM) results indicate that successful deposition of conjugated nanoparticles. And the primary umbilical vein endothelial cells would be applied to grow on the deposited substrate surface. Part of the substrate was covered among the deposition process. The primary umbilical vein endothelial cells were used to test the deposition result of $\operatorname{IgG}$ conjugated up-conversion nanoparticles. 

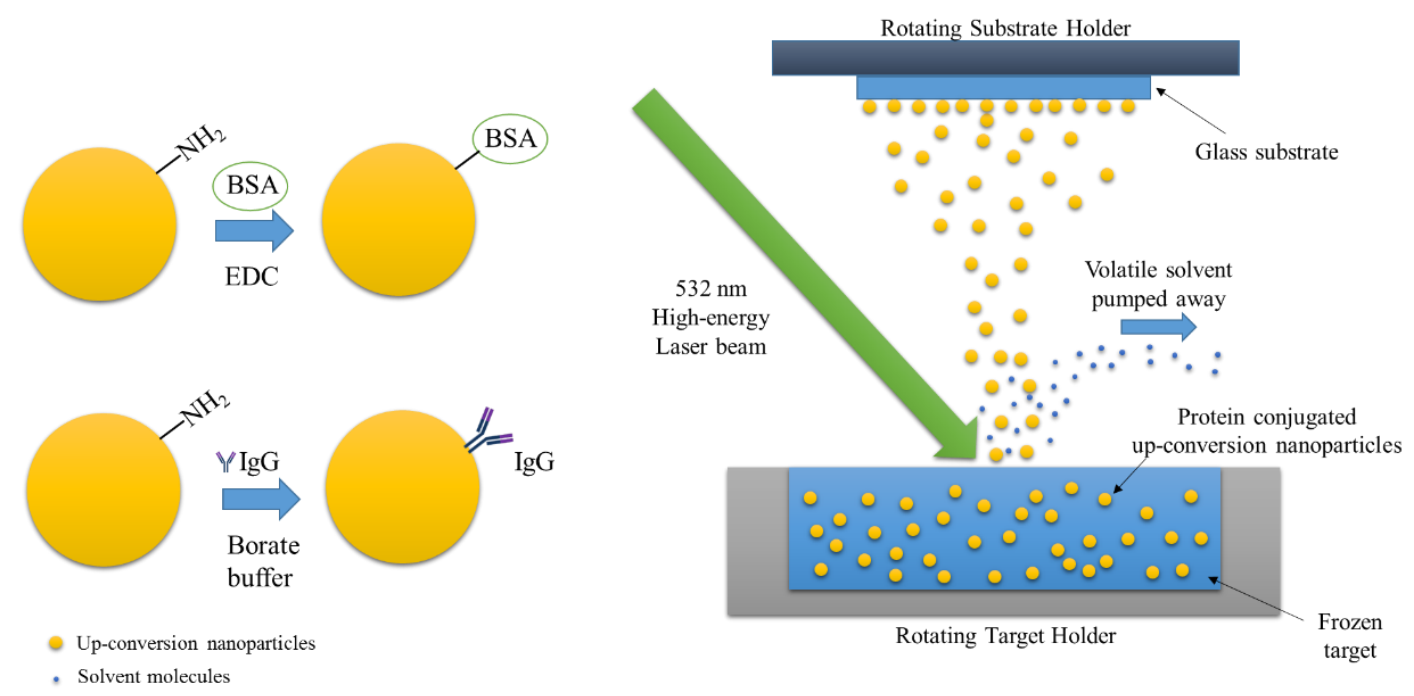

Fig. 1: Sketch of the conjugation process (BSA/IgG with up-conversion nanoparticles) and MAPLE deposition process.

\section{Materials and Experimental}

\subsection{Materials}

Gadolinium (III) nitrate hexahydrate (crystals and lumps, $99.9 \%$ trace metals basis), Erbium (III) nitrate pentahydrate (99.9\% trace metals basis), Ytterbium (III) nitrate pentahydrate (99.9\% trace metals basis),Bovine serum albumin (heat shock fraction, protease free, suitable for hybridization, $\mathrm{pH} 7, \geq 98 \%$ ), Anti-human IgG (fab-specific) FITC, N-(3Dimethylaminopropyl)-N'-ethylcarbodiimide hydrochloride (EDC, purum, $\geq 98.0 \%$ ), were purchased from Sigma-Aldrich. 2-propanol (Reagent grade) was purchased from Caledon Laboratory Chemicals. MAPLE equipment (MAPLE 2000, Project 206, PVD Products, Inc., USA) was applied. The water used in experiments were of $18.2 \mathrm{M} \Omega$ resistivity provided by Barnstead Water Purification System.

\subsection{Synthesis of $\mathrm{NaGdF}_{4}: \mathrm{Yb}: \mathrm{Er}$ Up-Conversion Nanoparticles}

The synthesis of up-conversion nanoparticles was based on this reference[13]. $720 \mathrm{mg}$ of Gadolinium (III) nitrate hexahydrate, $170 \mathrm{mg}$ of Erbium (III) nitrate pentahydrate and $160 \mathrm{mg}$ of Ytterbium (III) nitrate pentahydrate were added in $20 \mathrm{ml}$ ethylene glycol. Magnetic stirrer was applied to guarantee proper mixing of the solute. $0.7 \mathrm{~g}$ of branched polyethylenimine (PEI) were gently added to achieve proper dissolving since the viscosity of this agent is high. The dispersion was stirred at room temperature for approximately $60 \mathrm{~min}$.

$336 \mathrm{mg}$ Sodium fluoride were dissolved in $10 \mathrm{ml}$ ethylene glycol and added into dispersion dropwise. Then the dispersion was heated to $200{ }^{\circ} \mathrm{C}$ and refluxed for 6 hours. During the reaction process, nitrogen protection was applied. The products were collected by centrifugation and washed three times with ethanol and water. To collect dry powder of nanoparticles, the products were dissolve in $10 \mathrm{ml}$ ethanol and placed in a $37^{\circ} \mathrm{C}$ incubator for 12 hours.

\subsection{Preparation of the BSA Conjugated Up-Conversion Nanoparticles}

Since branched polyethyleneimine (PEI) was applied as the ligand of the up-conversion nanoparticles and the particle surface was covered with amino-group, the 1-Ethyl-3-(3-dimethylaminopropyl) carbodimide (EDC) was used to conjugate the BSA on the surface of up-conversion nanoparticles. Base on the reference[14], the obtained up-conversion nanoparticles were dissolve in water with a concentration of $2 \mathrm{mg} / \mathrm{ml}$. To conjugate BSA with up-conversion nanoparticles, $100 \mathrm{mg}$ of BSA powder was added to the $5 \mathrm{ml}$ as prepared nanoparticle solution and then $5 \mathrm{mg}$ EDC powder was added. This solution was stirred under ambient temperature for 2 hours. Then $5 \mathrm{mg}$ EDC was dissolve in the solution. The solution was further stirred for 6 hours under room temperature. The obtained BSA conjugated up-conversion nanoparticles were isolated by centrifugation. The products were re-dispersed in distilled water and stored under $4{ }^{\circ} \mathrm{C}$ environment. 


\subsection{Preparation of the IgG Conjugated Up-Conversion Nanoparticles}

The fabrication of $\mathrm{IgG}$ conjugated up-conversion nanoparticles base on this procedure. $15 \mathrm{mg}$ of as prepared upconversion nanoparticles was dissolved in $15 \mathrm{ml}$ water. After that $5 \mathrm{ml}$ borate buffered saline $(\mathrm{pH}=8)$ and $20 \mu \mathrm{g}$ of $\mathrm{IgG}$ was added to the solution. The solution was shaken under room temperature for 2 hours. The IgG conjugated upconversion nanoparticles were collected by centrifugation. The products were re-dispersed in distilled water and stored under $4{ }^{\circ} \mathrm{C}$ environment.

\subsection{The MAPLE Deposition Process of as Prepared Nanoparticles}

The as prepared nanoparticles were dispersed by the 2-propanol with a concentration of $1 \mathrm{wt}$. \%. The $2 \mathrm{ml}$ of dispersion was sonicated for 5 minutes to make the nanoparticles disperse uniformly. No other additional additives and surfactants were used. And the liquid nitrogen was used to freeze the target dispersion. The laser source which was used for the deposition are Nd:YAG laser (wavelength $532 \mathrm{~nm}$ ). The frequency of laser was $10 \mathrm{~Hz}$ and the $\tau_{\text {fwhm }} \cong 200 \mu \mathrm{s}$. The laser fluence was $120 \mathrm{~mJ} / \mathrm{cm}^{2}$ and the laser spot size was $0.56 \mathrm{~cm}^{2}$. The MAPLE deposition continued for 2 hours.

The substrate was a glass slip. During the deposition process the one third of substrate was covered and the temperature of the substrate was kept around $25^{\circ} \mathrm{C}$. The dispersion were immediately added to the target holder after the sonication treatment and frozen by the liquid nitrogen which flowed through the sidewall of the special target holder. Target dispersion were keep frozen during the deposition process. The experiment was conducted under $1 \times 10^{-6}$ Torr. The target and the substrate were rotating (target: $10 \mathrm{rpm}$, substrate: $25 \mathrm{rpm}$ ) during the laser irradiation to achieve a uniform morphology of the deposition on the substrate surface.

\subsection{Characterization}

TEM image was obtained by Philips CM-10 transmission electron microscope operating at $80 \mathrm{kV}$. Fourier transform infrared spectroscopy (FT-IR) was measured by Bruker Vector 22 FTIR spectrometer.

\section{Result and Discussions}

The TEM micrographs of up-conversion nanoparticles and BSA conjugated up-conversion nanoparticles was presented in Fig 2. The TEM indicate that the up-conversion nanoparticles were mono-dispersed and the average diameter around 50-60 nm with a cubic shape. Compare with the original up-conversion nanoparticles, a $4 \mathrm{~nm}$ BSA coating on the surface was clearly presented in the TEM micrograph. This indicated the BSA was successfully conjugated with the upconversion nanoparticles.
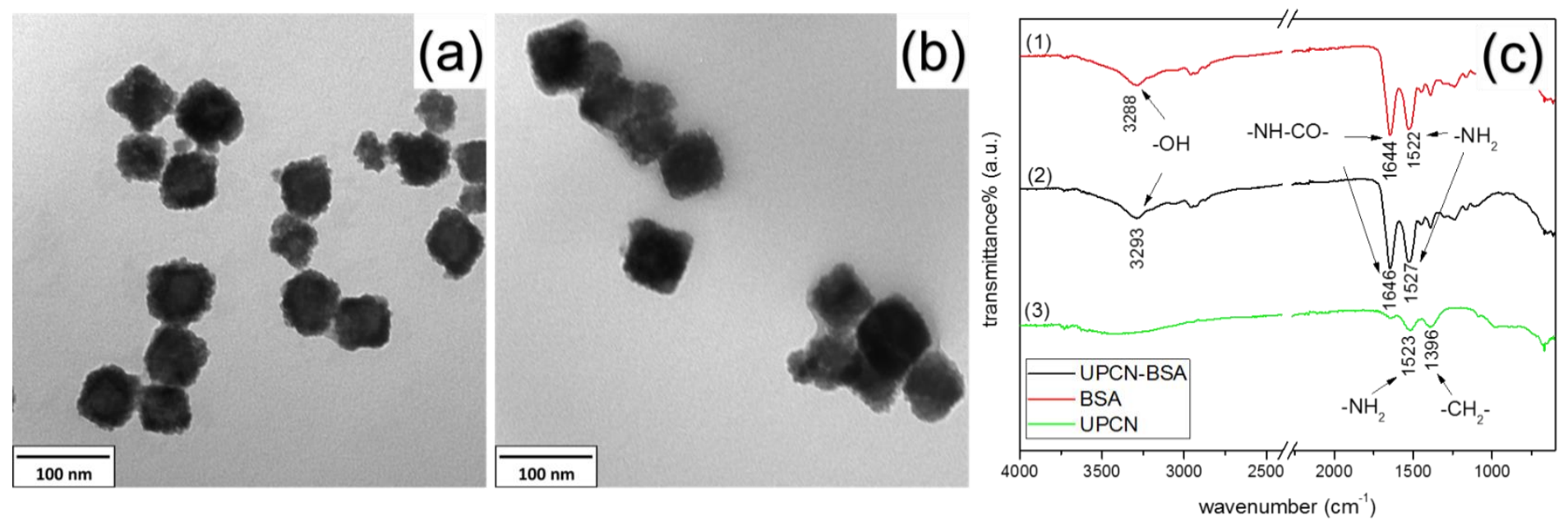

Fig. 2: The TEM micrograph of (a) up-conversion nanoparticles and (b) BSA conjugated up-conversion nanoparticles (c) FTIR spectra of (1) BSA powder (2) BSA conjugated up-conversion nanoparticles (3) up-conversion nanoparticles.

To further confirm the conjugation results, Fourier transform infrared spectroscopy (FT-IR) was applied (Fig. 2 c). The FT-IR result indicate BSA and BSA conjugated up-conversion nanoparticles showed stretching vibration band of the - 
$\mathrm{OH}$ group at $3288 \mathrm{~cm}^{-1}$ and $3293 \mathrm{~cm}^{-1}$. The $-\mathrm{OH}$ band of the up-conversion particles was not obvious since the particles were covered with branched PEI. The stretching vibrations band of the Carbonyl group was found at 1644 $\mathrm{cm}^{-1}$ and $1646 \mathrm{~cm}^{-1}$ for BSA and BSA conjugated up-conversion nanoparticles which suggest the present of the peptide bond and this stretching vibrations band was obvious compare with the original up-conversion spectrum. Besides, the stretching vibrations band of $-\mathrm{NH}_{2}$ was found at $1522 \mathrm{~cm}^{-1}, 1527 \mathrm{~cm}^{-1}$ and $1523 \mathrm{~cm}^{-1}$ in all 3 spectra because all these 3 materials contain amino group. Therefore, the successful conjugation was confirmed by the FT-IR spectra.

The TEM micrographs of the up-conversion nanoparticles and BSA conjugated up-conversion nanoparticles were also presented in Fig 3 (a) and Fig 3 (b). The TEM micrograph of the up-conversion nanoparticles on the substrate indicate the size was $77 \mathrm{~nm}$ and it were not changed obviously. However the shape of the up-conversion may be influenced by the laser and changed from cubic shape to sphere shape. Fig 4 (b) is the TEM micrograph of the BSA conjugated up-conversion nanoparticles which were deposited on the substrate[15]. The BSA coating on the surface of the nanoparticle was obvious in this micrograph. The size of the nanoparticles were around $50 \mathrm{~nm}$ and particles were sphere shape and cubic shape. This confirm the successful deposition of the up-conversion particles and BSA conjugated up-conversion nanoparticles on the glass substrate through the MAPLE technique. Beside this result indicate that the laser effect were minimized by the volatile solvent.
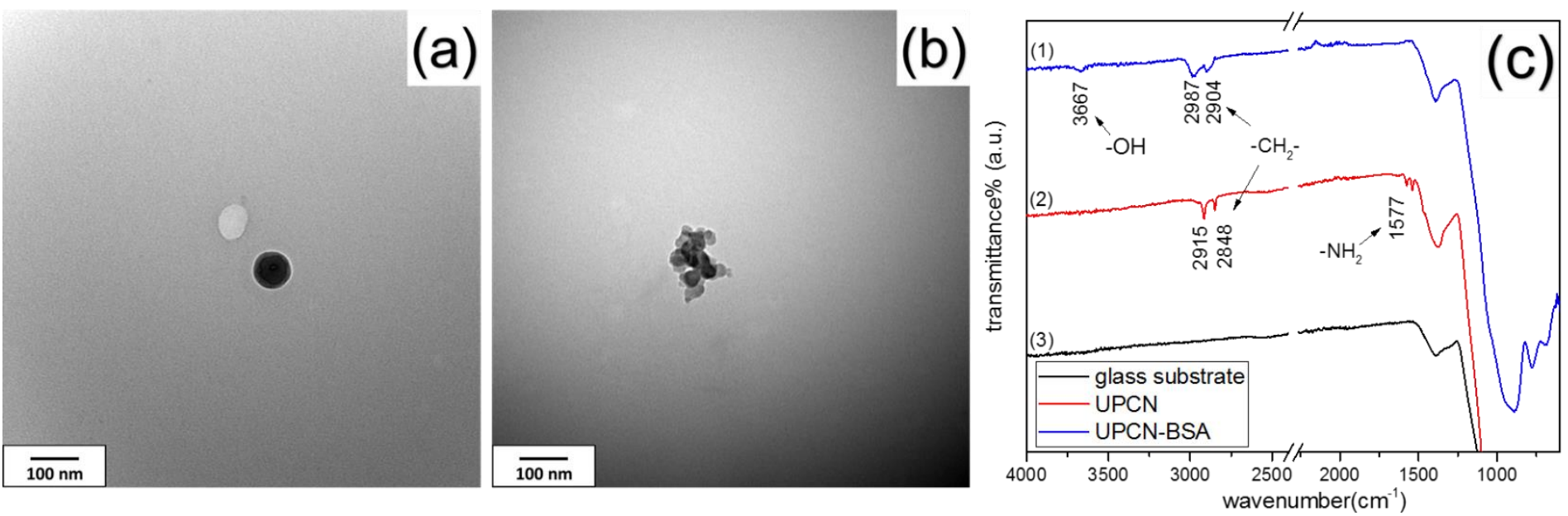

Fig. 3: The TEM micrograph of (a) up-conversion nanoparticles and (b) BSA conjugated up-conversion nanoparticles on the substrate after MAPLE deposition (c) FTIR spectra of (1) BSA conjugated up-conversion nanoparticles deposited on glass substrate (2) upconversion nanoparticles deposited glass substrate (3) glass substrate.

The FT-IR characterization (Fig 3 c) was applied to further confirm the deposition result of the up-conversion nanoparticles and BSA conjugated up-conversion nanoparticles. The stretching vibration band of $-\mathrm{OH}$ group was found at $3667 \mathrm{~cm}-1$ this indicate the existence of carboxyl group on the substrate which was deposited BSA-conjugated upconversion nanoparticles on its surface. The stretching vibration band of $-\mathrm{NH} 2$ was found at $1577 \mathrm{~cm}-1$ and this due to the amino group which was fixed on the surface of up-conversion nanoparticles. And the stretching vibration band of methylene could be found in both two spectra, lead to the existence of BSA and branched PEI. Therefore, the FT-IR spectra confirmed the successful deposition of both up-conversion nanoparticles and BSA conjugated up-conversion nanoparticles.

Then the IgG conjugated up-conversion nanoparticles were deposited on the glass slide. And substrate were test with FT-IR after treated with MAPLE deposition process (Fig 4). The result indicate that the stretching vibration band of $-\mathrm{OH}$ group could be confirmed at $3648 \mathrm{~cm}-1$. And the existence of methylene could be confirmed by the stretching vibration band at $2985 \mathrm{~cm}-1$ and $2900 \mathrm{~cm}-1$. Compare with the spectrum of the up-conversion nanoparticles and glass substrate, this result could confirm the existence of IgG conjugated up-conversion nanoparticles on the substrate surface after MAPLE deposition. 


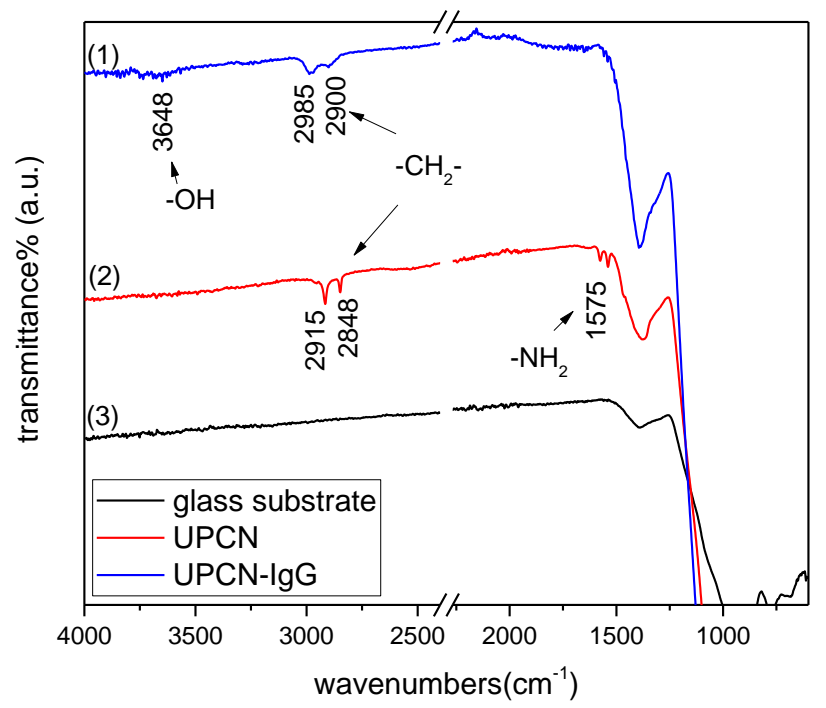

Fig. 4: FTIR spectra of (1) IgG conjugated up-conversion nanoparticles deposited on glass substrate (2) up-conversion nanoparticles deposited glass substrate (3) glass substrate.

\section{Conclusion}

In this study, the up-conversion nanoparticles, BSA conjugated up-conversion nanoparticles and IgG conjugated nanoparticles were successfully deposited on the surface of the glass substrate. The TEM and FT-IR result confirmed the immobilization of the these nanoparticles which were achieved by the MAPLE equipment with a pulsed Nd:YAG laser at $532 \mathrm{~nm}$. Besides, the influence of laser to the shape and size of the up-conversion nanoparticles were minimized. The BSA and IgG was protected by the volatile solvent and deposited on the surface of the glass substrate along with the upconversion nanoparticles. Our group are currently working on developing biomaterial device based on this result and test the interaction between the deposited $\mathrm{BSA} / \mathrm{IgG}$ up-conversion nanoparticles with primary umbilical vein endothelial cells.

\section{References}

[1] F. Auzel, "Upconversion and Anti-Stokes Processes with $\mathrm{f}$ and d Ions in Solids," Chem. Rev., vol. 104, no. 1, pp. 139-174, 2004.

[2] M. Haase and H. Schäfer, "Upconverting Nanoparticles," Angewandte Chemie International Edition, vol. 50, no. 26, pp. 5808-5829, 2011.

[3] X. Liu, C.-H. Yan, and J. A. Capobianco, "Photon upconversion nanomaterials," Chem. Soc. Rev., vol. 44, no. 6, pp. 1299-1301, 2015.

[4] C. Li, J. Liu, S. Alonso, F. Li, and Y. Zhang, "Upconversion nanoparticles for sensitive and in-depth detection of $\mathrm{Cu}^{2+}$ ions," Nanoscale, vol. 4, no. 19, pp. 6065-6071, 2012.

[5] J. Zhou, Z. Liu, and F. Li, "Upconversion nanophosphors for small-animal imaging," Chem. Soc. Rev., vol. 41, no. 3, pp. 1323-1349, 2012.

[6] K. Liu, X. Liu, Q. Zeng, Y. Zhang, L. Tu, T. Liu, et al, "Covalently Assembled NIR Nanoplatform for Simultaneous Fluorescence Imaging and Photodynamic Therapy of Cancer Cells," ACS Nano, vol. 6, no. 5, pp. 4054-4062, 2012.

[7] G. Tian, W. Ren, L. Yan, S. Jian, Z. Gu, L. Zhou, et al, "Red-Emitting Upconverting Nanoparticles for Photodynamic Therapy in Cancer Cells Under Near-Infrared Excitation," Small, vol. 9, no. 11, pp. 1929-1938, 2013.

[8] H. J. M. A. A. Zijlmans, J. Bonnet, J. Burton, K. Kardos, T. Vail, R. S. Niedbala, et al, "Detection of Cell and Tissue Surface Antigens Using Up-Converting Phosphors: A New Reporter Technology," Anal. Biochem., vol. 267, no. 1, pp. 30-36, 1999.

[9] D. J. Gargas, E. M. Chan, A. D. Ostrowski, S. Aloni, M. V. P. Altoe, E. S. Barnard, et al, "Engineering bright sub10-nm upconverting nanocrystals for single-molecule imaging," Nat Nano, vol. 9, no. 4, pp. 300-305, 2014. 
[10] L. Cheng, K. Yang, Y. Li, J. Chen, C. Wang, M. Shao, et al, "Facile Preparation of Multifunctional Upconversion Nanoprobes for Multimodal Imaging and Dual-Targeted Photothermal Therapy," Angew. Chem., vol. 123, no. 32, pp. 7523-7528, 2011.

[11] D. B. Chrisey, A. Piqué, R. A. McGill, J. S. Horwitz, B. R. Ringeisen, D. M. Bubb, et al, "Laser Deposition of Polymer and Biomaterial Films," Chem. Rev., vol. 103, no. 2, pp. 553-576, 2003.

[12] M. Verrastro, N. Cicco, F. Crispo, A. Morone, M. Dinescu, M. Dumitru, et al, "Amperometric biosensor based on Laccase immobilized onto a screen-printed electrode by Matrix Assisted Pulsed Laser Evaporation," Talanta, vol. 154, pp. 438-445, 2016.

[13] H.-T. Wong, M.-K. Tsang, C.-F. Chan, K.-L. Wong, B. Fei, and J. Hao, "In vitro cell imaging using multifunctional small sized $\mathrm{KGdF}_{4}: \mathrm{Yb}^{3+}, \mathrm{Er}^{3+}$ upconverting nanoparticles synthesized by a one-pot solvothermal process," Nanoscale, vol. 5, no. 8, pp. 3465-3473, 2013.

[14] Q. Chen, C. Wang, L. Cheng, W. He, Z. Cheng, and Z. Liu, "Protein modified upconversion nanoparticles for imaging-guided combined photothermal and photodynamic therapy," Biomaterials, vol. 35, no. 9, pp. 2915-2923, 2014.

[15] A. P. Caricato, V. Arima, M. Catalano, M. Cesaria, P. D. Cozzoli, M. Martino, et al, "MAPLE deposition of nanomaterials," Appl. Surf. Sci., vol. 302, pp. 92-98, 2014. 